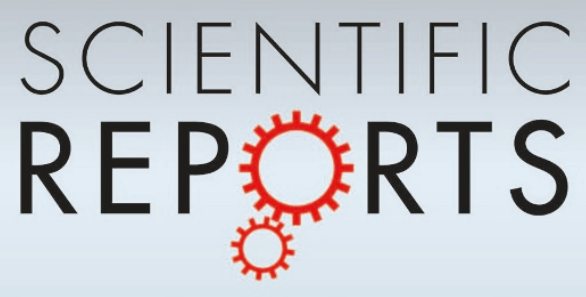

OPEN

SUBJECT AREAS:

MODELLING

VIROLOGY

NEURODEGENERATION

MOLECULAR ENGINEERING

Received

3 January 2012

Accepted

19 January 2012

Published

9 February 2012

Correspondence and requests for materials should be addressed to

E.B. (erwan.bezard@ u-bordeaux2.fr)

\section{Systemic scAAV9 variant mediates brain transduction in newborn rhesus} macaques

\author{
Benjamin Dehay ${ }^{1,2}$, Deniz Dalkara ${ }^{3}$, Sandra Dovero ${ }^{1,2}$, Qin Li4 \& Erwan Bezard',2,4
}

\begin{abstract}
'Univ. de Bordeaux, Institut des Maladies Neurodégénératives, UMR 5293, F-33000 Bordeaux, France, ${ }^{2} \mathrm{CNRS}$, Institut des Maladies Neurodégénératives, UMR 5293, F-33000 Bordeaux, France, ${ }^{3}$ Department of Chemical Engineering, Department of Bioengineering, and The Helen Wills Neuroscience Institute, The University of California, Berkeley, CA 94720, ${ }^{4}$ Institute of Lab Animal Sciences, China Academy of Medical Sciences, Beijing, China.
\end{abstract}

Transgenic macaques would allow to study brain function and diseases. We report that an engineered adeno-associated virus serotype 9 variant (scAAV9) injected intravenously in newborn rhesus macaques results in efficient, exclusively-neuronal and widespread transduction of the brain. The present data pave the way to large-scale genetic modelling of brain diseases in the rhesus macaque.

T he initial advancements in transgenic technology have led to the generation of the first transgenic monkey in $2001^{1}$ and a transgenic monkey model of Huntington's disease in $2008^{2}$. These breakthroughs have opened the door to genetic manipulation in nonhuman primates but the technical difficulties have prevented this long awaited technological advancement for the study of brain function to be further developed ${ }^{3}$. The recent development of the viral-mediated delivery of genes has led to the identification of viruses capable of transducing developing cells and neurons $s^{4-5}$. Adeno-associated virus (AAV) vectors can mediate long-term stable transduction in various target tissues. Among those, AAV serotype 9 (AAV9) vectors possess unique characteristics with notably a striking ability to cross the blood brain barrier ${ }^{5}$. After intraparenchymal injection, AAV9 is more readily transported within the brain compared with other AAV serotypes ${ }^{6}$. Given the unique properties of AAV9 ${ }^{6}$, this serotype was tested for delivering genes to the central nervous system after intravenous injection in many species. When i.v. injected in adult mice, transduction occurs predominantly in astrocytes in both spinal cord and brain. However, when injected to neonate mice or newborn monkeys, a single intravascular AAV9 injection led to extensive transduction (60\%) in dorsal root ganglia, motor neurons and in neurons in most mice brain regions ${ }^{4,7}$. However, not a single study described precisely the brain tropism of AAV9 vectors administrated intravenously in non-human primates. AAV9 therapy has recently lead to proof-of-concept preclinical rescues in mouse models (i) of spinal muscular atrophy disease ${ }^{8-9}$ and (ii) of a lysosomal storage disorder ${ }^{10}$. Ability to transfect brain decreases over time, being high at P1 and decreasing dramatically by P10 already ${ }^{8}$ suggesting a developmental period in which AAV9 transfection has maximal efficacy.

We here investigated if transduction in the brain cells can be achieved using an engineered AAV9 vector in the rhesus macaque (macaca mulatta), the species of choice in neuroscience in general and in biological psychiatry in particular.

\section{Results}

GFP expression was found in all analyzed regions, including the cortex, striatum, globus pallidus, thalamus, midbrain, hippocampus and cerebellum (Fig. 1B-J). GFP-positive cells were more numerous in regions known to undergo profound post-natal maturation, such as cortex, hippocampus and Purkinje cells in the cerebellum (Fig. 2A). A quantitative unbiased stereological evaluation of the number of GFP-transduced cell confirmed these observations (Fig. 2B). Even the newborn monkey injected with the lowest viral titer exhibited an efficient neuronal transduction (Fig. 2B). Posited tropism towards neurons among brain cells was confirmed using triple staining for NeuN to label neurons, DAPI to label cell nuclei, and anti-GFP antibodies to label transduced-cells. All GFP-positive cells were NeuN-positive and no GFP-positive glial cell was observed, thereby validating the neuronal specificity of transduction in macaques injected at post-natal day 1 (Fig. 2C). Finally, to evaluate distribution to other organs, sections of the liver, the heart, the muscles, the lungs and the kidneys were analyzed 


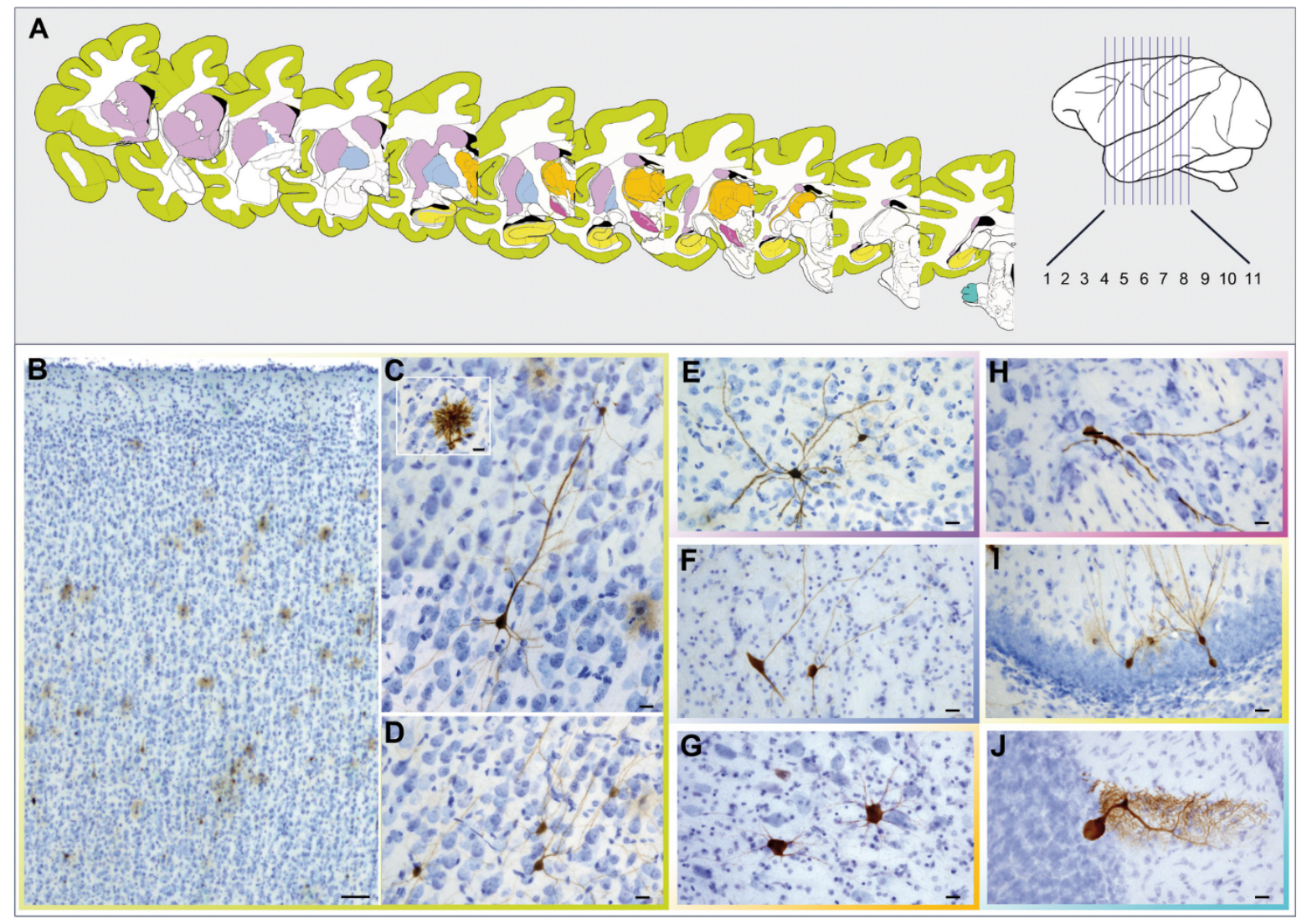

Figure 1 Neuronal cell-type specificity and distribution resulting from AAV9 injections at day 1 post-natal. (A) Rostro-caudal representation of observed sections after intravenous injection of AAV9-CMVie-GFP. Color code relates highlighted areas to color frames. (B-D) Extensive GFP expression within cortical neurons was observed (B), including layer II/III pyramidal cell (inset: cortical spiny stellate cell are star-shaped) (C) and layer IV spiny stellate cell (D). Neurons were easily detected in the striatum (left: medium spiny neurons; right: small striatal neurons) (E), globus pallidus (F), thalamus (G), substantia nigra (H), hippocampus (I) and cerebellum (J). Scale bars, $100 \mu \mathrm{m}(\mathrm{B}) ; 20 \mu \mathrm{m}(\mathrm{C}-\mathrm{J})$.

A

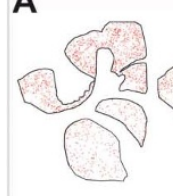

$-\quad A^{A C+2}$

C
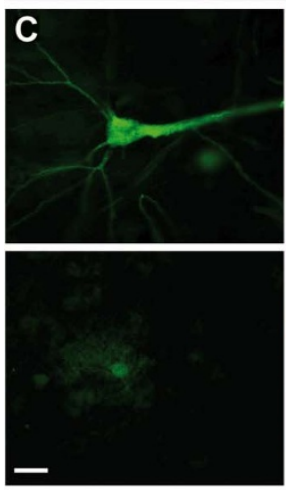

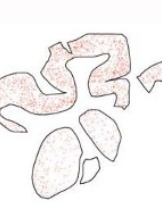

AC 0

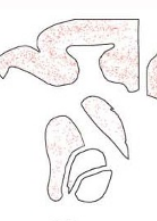

AC-2
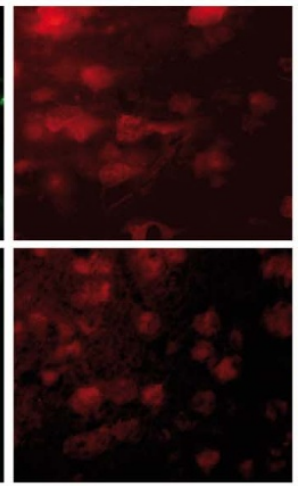

B
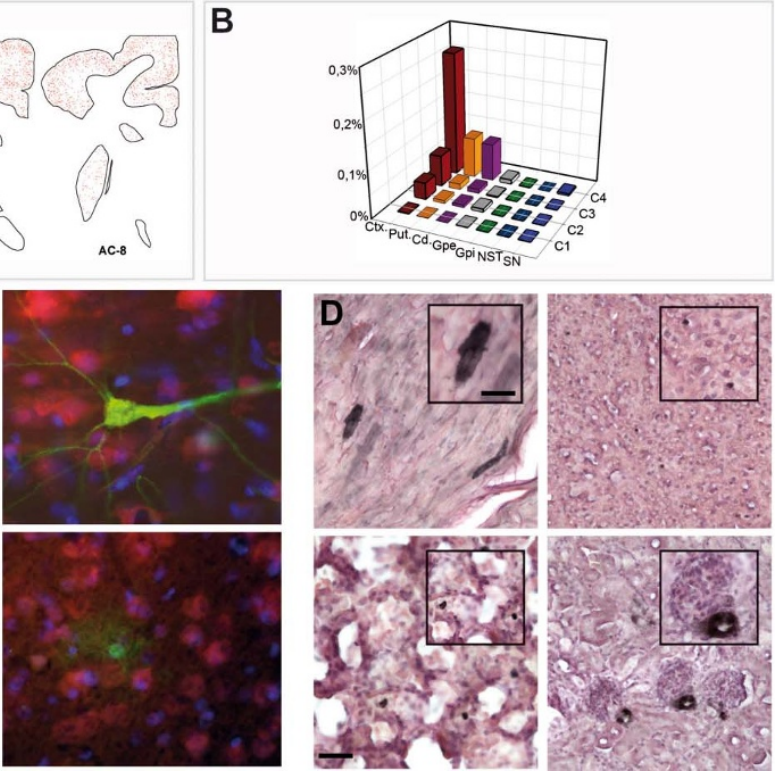
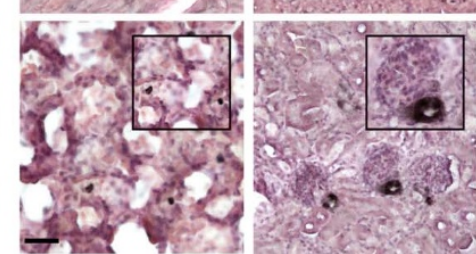

Figure $2 \mid$ Broad transduction of the nervous system and peripheral organs resulting from AAV9 injections at day 1 post-natal. (A) Rostro-caudal representation of GFP-transduced neurons after intravenous injection of AAV9-CMVie-GFP. Red dots relate GFP-positive cells. (B) Quantitative analysis of GFP-positive cells in all analyzed regions, including the cortex, caudate, putamen, globus pallidus internal and external, subthalamic nucleus and substantia nigra in four newborn monkeys. (C) To confirm transduction of neurons, sections of cortex from newborn injected monkeys were immunofluorescently labeled with anti-GFP antibody (green), neuron-specific NeuN antibody (red). Merging of the signals produced colocalization, thus confirming neuronal transduction. (D) Representative images from different organs from C4 monkey (Top left: heart; Top right: liver; Bottom Left: lung; Bottom right: kidney). Scale bars, $2000 \mu \mathrm{m}$ (A); $20 \mu \mathrm{m}$ (C); $80 \mu \mathrm{m}$ (D); $20 \mu \mathrm{m}$ (inset D). 
for GFP immunopositivity. Neonatal administration of AAV9 resulted in few GFP-positive cells within heart, liver, lungs and kidneys (Fig. 2D) while no GFP signal was detected in the muscles.

\section{Discussion}

Althogether, our results show that intravenous delivery of AAV9GFP at a dose range of $10^{12}-10^{14}$ genome copies in one day-old rhesus macaque results in widespread neuronal targeting with the maximum transduction efficiency at a viral titer of $10^{12} \mathrm{vg} / \mathrm{ml}$. Transduction of neurons was observed in most regions of the brain with some heterogeneity, the post-nataly developing structures like the cortex and hippocampus being more transfected than deep brain structures such as the midbrain. Although AAV9 appears a robust viral vector for gene transfer to the brain, it would provide a promising research tool for delivering genes in nonhuman primate only if we could reach all structures with comparable efficiency, the specificity of expression being driven by structure-specific promoters (i.e. tyrosine hydroxylase for dopaminergic neurons, parvalbumin for GABAergic interneurons, etc.). Recent developments of in utero gene delivery technologies has clearly demonstrated that a near total transfection of neurons is possible at embryonic stages compatible with the development of the targeted area ${ }^{11-12}$ and such route needs to be now considered for non-human primates. Additionally, other viral engineering approaches can be used to further enhance the transduction capabilities of AAV in this context ${ }^{13-14}$. The present data thus pave the way to the genetic modeling of brain diseases in the rhesus macaque by targeting mutant gene with tissue-specific gene expression, offering unique opportunities for modeling human disease onset, progression and for validating therapeutic solutions.

\section{Methods}

Animals were housed in social cages with their mothers under controlled conditions of humidity, temperature, and light (12-h light/12-h dark cycle, lights on at $8.00 \mathrm{am}$ ); food and water were available ad libitum. Experiments were carried out in accordance with European Communities Council Directive of 3 June 2010 (2010/6106/EU) for care of laboratory animals in an AAALAC-accredited facility following acceptance of study design by the Institute of Lab Animal Science (Chinese Academy of Science, Beijing, China) IACUC. One day-old male rhesus macaques received intravenous injections of an AAV9 vector that expresses green fluorescent protein (GFP) under the control of cytomegalovirus immediate early synapsin intron promoter (AAV9CMVie-GFP). Four newborn male monkeys (C1 to $\mathrm{C} 4)$ were injected with $1 \mathrm{ml}$ with 4 different AAV9 viral titers $\left(\mathrm{C} 1: 4.00 \times 10^{14} \mathrm{vg} / \mathrm{ml}\left[1.33 \times 10^{12}\right.\right.$ particles $/ \mathrm{g}$ of body weight]; $C 2: 9.61 \times 10^{13} \mathrm{vg} / \mathrm{ml}\left[3.2 \times 10^{11}\right.$ particles/g of body weight $]$; C3: $2.65 \times 10^{14} \mathrm{vg} / \mathrm{ml}\left[8.83 \times 10^{11}\right.$ particles $/ \mathrm{g}$ of body weight]; C4: $2.85 \times 10^{12} \mathrm{vg} / \mathrm{ml}$ $\left[9.5 \times 10^{9}\right.$ particles/g of body weight $]$ ). Animals were longitudinally followed and euthanized 2 months post-injection ${ }^{15}$. GFP immunohistochemistry was performed along the rostro-caudal axis of the brain serially cut on a cryostat (50 $\mu \mathrm{m}$-thick; Fig. 1A $)^{15}$. Double immunostaining procedure against GFP and NeuN were performed sequentially. Briefly, $50 \mu \mathrm{m}$ free floating sections were first incubated in a mouse NeuN antibody (Millipore, MAB377) diluted to $1 / 500$ in PBS-BSA (1/50)Triton $0.3 \% \mathrm{O} / \mathrm{N}$ at room temperature (RT). After several washes, NeuN signal was revealed by incubating a goat anti-mouse antibody conjugated to Alexa568 (Invitrogen, A11004) for $1 \mathrm{hr}$ at RT. Sections were then incubated with rabbit antiGFP antibody (Invitrogen, A11122) diluted to 1/1000 in PBS-BSA (1/50)-Triton 0.3\% for $2 \mathrm{hr}$ at $37^{\circ} \mathrm{C}$, washed with PBS and revealed with donkey anti-rabbit antibody conjugated to Alexa 488 (Invitrogen, A21206) for $1 \mathrm{hr}$ at RT. DAPI staining was performed using Hoechst dye (Invitrogen, H3569) at $6 \mu \mathrm{g} / \mathrm{mL}$ in PBS for $2 \mathrm{~min}$.
1. Chan, A. W., Chong, K. Y., Martinovich, C., Simerly, C. \& Schatten, G. Transgenic monkeys produced by retroviral gene transfer into mature oocytes. Science $\mathbf{2 9 1}$ 309-312 (2001)

2. Yang, S. H. et al. Towards a transgenic model of Huntington's disease in a nonhuman primate. Nature 453, 921-924 (2008).

3. Bowers, W. J., Breakefield, X. O. \& Sena-Esteves, M. Genetic therapy for the nervous system. Hum Mol Genet 20, R28-41 (2011).

4. Foust, K. D. et al. Intravascular AAV9 preferentially targets neonatal neurons and adult astrocytes. Nat Biotechnol 27, 59-65 (2009).

5. Zhang, H. et al. Several rAAV vectors efficiently cross the blood-brain barrier and transduce neurons and astrocytes in the neonatal mouse central nervous system. Mol Ther 19, 1440-1448 (2011).

6. Cearley, C. N. \& Wolfe, J. H. Transduction characteristics of adeno-associated virus vectors expressing cap serotypes 7, 8, 9, and Rh10 in the mouse brain. Mol Ther 13, 528-537 (2006).

7. Duque, S. et al. Intravenous administration of self-complementary AAV9 enables transgene delivery to adult motor neurons. Mol Ther 17, 1187-1196 (2009).

8. Foust, K. D. et al. Rescue of the spinal muscular atrophy phenotype in a mouse model by early postnatal delivery of SMN. Nat Biotechnol 28, 271-274 (2010).

9. Dominguez, E. et al. Intravenous scAAV9 delivery of a codon-optimized SMN1 sequence rescues SMA mice. Hum Mol Genet 20, 681-693 (2011).

10. Spampanato, C. et al. Efficacy of a combined intracerebral and systemic gene delivery approach for the treatment of a severe lysosomal storage disorder. $\mathrm{Mol}$ Ther 19, 860-869 (2011).

11. Kamiya, A. Animal models for schizophrenia via in utero gene transfer: understanding roles for genetic susceptibility factors in brain development. Prog Brain Res 179, 9-15 (2009).

12. Rahim, A. A. et al. Intravenous administration of AAV2/9 to the fetal and neonatal mouse leads to differential targeting of CNS cell types and extensive transduction of the nervous system. FASEB J 25, 3505-3518 (2011).

13. Gray, S. J. et al. Directed evolution of a novel adeno-associated virus (AAV) vector that crosses the seizure-compromised blood-brain barrier (BBB). Mol Ther 18, 570-578 (2010)

14. Maheshri, N., Koerber, J. T., Kaspar, B. K. \& Schaffer, D. V. Directed evolution of adeno-associated virus yields enhanced gene delivery vectors. Nat Biotechnol 24, 198-204 (2006).

15. Fasano, S. et al. Inhibition of Ras-guanine nucleotide-releasing factor 1 (Ras-GRF1) signaling in the striatum reverts motor symptoms associated with L-dopa-induced dyskinesia. Proc Natl Acad Sci U S A 107, 21824-21829 (2010).

\section{Acknowledgments}

This work was supported by European Commission's Marie Curie Reintegration Grant (FP7-PEOPLE-2009-ERG256303, to B.D.), Fondation pour la Recherche Médicale Grant (BD) and by Agence Nationale de la Recherche grants (EB: ANR-08-MNP-018 MCHPRIMAPARK). We thank Audrey Martinez for her tremendous technical support. The Université Bordeaux Segalen and the Centre National de la Recherche Scientifique provided the infrastructural support.

\section{Author contribution statement}

B.D. and E.B. designed research; B.D., D.D., S.D., Q.L. performed research; B.D., S.D. and E.B. analyzed data; B.D. and E.B. wrote the main manuscript text and S.D. prepared figures 1-2. All authors reviewed the manuscript.

\section{Additional information}

Competing financial interests: EB is Chief Scientific Officer of Motac neuroscience Ltd. All other authors reported no biomedical financial interests or potential conflicts of interest.

License: This work is licensed under a Creative Commons Attribution-NonCommercialNoDerivative Works 3.0 Unported License. To view a copy of this license, visit http:// creativecommons.org/licenses/by-nc-nd/3.0/

How to cite this article: Dehay, B., Dalkara, D., Dovero, S., Li, Q. \& Bezard, E. Systemic scAAV9 variant mediates brain transduction in newborn rhesus macaques. Sci. Rep. 2, 253; DOI:10.1038/srep00253 (2012). 